\title{
La intervención del trabajo social en las instituciones educativas de la región Junín
}

\section{The intervention of social work in the educational institutions of the Junín region}

\author{
'Fabián Arias Vda. de Rivera, Eugenia; Vilcas Baldeón, Luz María; Matos Maldonado, Nidia Gladys \\ Vilcapoma Flores, Nina Noris \\ Facultad de Trabajo Social, Universidad Nacional del Centro del Perú \\ Email: efabian@uncp.edu.pe
}

\section{Resumen}

El objetivo fue caracterizar la intervención del trabajo social en las instituciones educativas de la región Junín. El tipo de investigación es básico, del nivel descriptivo y de diseño no experimental, transaccional, descriptivo. Las unidades de análisis fueron trabajadoras sociales que laboran en las instituciones educativas estatales y privadas de la región Junín; la población fue de 15 trabajadoras sociales, la muestra fue la misma que la población. El instrumento fue el cuestionario validado con el coeficiente de concordancia de $\mathrm{V}$ de Aiken, en donde todos los indicadores del instrumento de medición presentan validez de 0.72 a 0.99 lo que indica validez de contenido y la técnica fue la encuesta.

Los resultados revelaron que, el $79 \%$ de las trabajadoras sociales manifestaron que el objeto de intervención en las instituciones educativas de la región Junín son los problemas y/o necesidades sociales de los estudiantes; el objetivo de intervención es la formación integral de los estudiantes. El 79 \% considera que las funciones más frecuentes son la asistencial (atención inmediata y otros) y administrativa. El 100 \% declaró que las formas de intervención más predominante son los grupales e individuales; además, el $80 \%$ consideró que el proceso de intervención es el diagnóstico, planificación, ejecución y evaluación de actividades. Se concluye que el trabajo social es predominantemente administrativo, asistencial y de educación social.

Palabras claves: intervención del trabajo social, objeto de trabajo, funciones.

\begin{abstract}
The objective was to characterize the intervention of social work in the educational institutions of the Junín region. The type is basic, of descriptive level, and non-experimental, transactional, descriptive design. The units of analysis were social workers labouring in public and private educational institutions and the sample was 15 female social workers, the sample was the same as the popullation. The instrument was the validated questionnaire using the coefficient of V agreement of Aiken, where all the indicators of the measuring instrument are valid from 0.72 to 0.99 which indicates the validity of the content and the technique was the survey.

The results revealed that $79 \%$ of social workers stated that the object of intervention in the educational institutions of the Junin region are the problems and/or social needs of the students; the objective of intervention is the integral formation of the students. $79 \%$ considers that the most frequent functions are the assistance (immediate attention and other) and administrative. $100 \%$ stated that the most predominant forms of intervention are group and individual, in addition, $80 \%$ considered that the intervention process is the diagnosis, planning, execution and evaluation of activities. The conclusion is that social work predominantly is administrative, assistance and social education.
\end{abstract}

Keywords: social work intervention, object of work, functions. 


\section{Introducción}

El trabajo social es una profesión, que en su proceso histórico a estado y está interrelacionado con el contexto mundial contemporáneo, el cual tiene como expresiones matrices la globalización económica, la alta conectividad entre los hechos y contextos locales y globales, la pobreza las inequidades y desigualdades sociales, la necesidad del reconocimiento de la pluriculturidad, el interés por las minorías y grupos poblacionales vulnerables que irrumpen como sujetos sociales de gran importancia y el resurgir de los derechos humanos como eje central de modelos de desarrollo que privilegian la dignidad y bienestar humano y preservan la vida en el planeta gracias a las sustentabilidad de la base natural del mismo. Maya (2008)

En este sentido, la carrera de Trabajo Social es una disciplina de las ciencias sociales que está orientado a contribuir en la solución de los problemas sociales, susceptibles de ser modificados por la intervención profesional.

Todas las profesiones intentan redefinir y asegurar las funciones y conocimientos especializados como fundamento de la realización personal. El concepto de función surge de la naturaleza misma del proceso social del trabajo, permitiendo identificar la aportación que hace a la sociedad una profesión, describiendo y estableciendo sus límites. Todas las profesiones encuentran sus razones de ser en las necesidades sociales y en toda colectividad existe la división de funciones entra personas o grupos, de modo que cada cual realice una contribución especifica al conjunto de la sociedad. Ballesteros, Viscarret y Uriz (2013).

Portuondo (1983), citado por Ballesteros, Viscarret y Uriz (2013), define la función como el tipo de actividad laboral que caracteriza el trabajo socialmente útil que realiza el hombre para alcanzar un objetivo determinado. Desde esta perspectiva la función comprende la esfera de responsabilidad que establecen las actividades relacionadas entre sí y encaminadas a un fin, determinando el ejercicio de una profesión. Estas deben reflejar el conjunto de problemas que las profesionales deben ser capaces de enfrentar, analizar y resolver. (Pág. 127)

Por consiguiente, las funciones de las trabajadoras sociales son; la función asistencial que responde a la atención de individuos o grupos que presentan o están en riesgo de presentar problemas de índole social; la función de planificación, considerado como la acción de ordenar o conducir un plan de acuerdo a objetivos determinados; la función de educación social, donde se realiza acciones orientadas a reflexionar y hacer partícipe en la solución de sus propios problemas y; la función de investigación, proceso metodológico que lleva a descubrir una realidad empleando técnicas con el fin de contextualizar una adecuada intervención.

En las ciencias sociales, continuamente se destaca la importancia de la confrontación de la teoría y la práctica, la comparación entre lo que conocemos y la realidad en donde aplicamos el saber constituye el núcleo y la razón de ser de los científicos de lo social, en este caso de la disciplina de Trabajo Social, ésta confrontación se produce en la intervención que realizan sus especialistas y una de sus formas básicas se da a través de la práctica de comunidad. Es aquí donde observamos y verificamos la validez del bagaje teórico que guía la intervención. Silva (1996). En este contexto, una de las prioridades de la investigación en el campo del trabajo social es el abordaje directo del problema de la relación entre teoría y practica; por consiguiente, el propósito es conocer si las trabajadoras sociales que laboran en el sector educación cumplen las funciones que se les enseña en las aulas universitarias de acuerdo al plan curricular o si en la práctica han ido cambiando o adaptándose a otras funciones o demandas que respondan a las necesidades del contexto. Los estudiantes de las instituciones educativas, afrontan una serie de problemas sociales, como violencia escolar, bullying, bajo rendimiento escolar, incorrectas relaciones entre compañeros, comunicación inadecuada, débil apoyo de los padres en las tareas escolares, problemas de desnutrición, desintegración familiar, separación de los padres, disfuncionalidad familiar, etc. En este contexto, la Facultad de Trabajo Social, interviene frente a dicha problemática mediante el proceso metodológico de investigación, diagnóstico, planificación, ejecución, monitoreo y evaluación; sin embargo, lo que se pretende conocer es si las trabajadoras sociales, que laboran en el sector educación, aplican el proceso metodológico mencionado y/o es otro el proceso que aplican.

Por otra parte, cabe resaltar que las funciones que cumplen no se dan a cabalidad y que su trascendencia es muy limitada; por las razones expuestas, se hace necesaria la investigación en esta área de intervención. Sobre el objeto de intervención del trabajo social en instituciones educativas, se comprende a las necesidades sociales factibles de ser modificados por la intervención en las instituciones educativas; en este sentido, se identifican problemáticas como violencia escolar, violencia intrafamiliar, dificultades en torno a principios y valores, consumo de sustancias psicoactivas, situaciones derivadas de las características de la educación sexual y reproductiva, etc., que afectan al desarrollo humano de la poblaciones de niños, niñas, adolescentes y familias.

La hipótesis general planteada fue: la intervención de la trabajadora social en las instituciones educativas de la región Junín es buena, porque cumple la función, gerencial, educación social, consejería - orientación social, seguido por la función de investigación; su objeto de intervención son los problemas y necesidades del estudiante; el objetivo es contribuir a la formación integral del estudiante, las formas de intervención predominante es el individual y grupal, aplicando el proceso de la investigación, diagnóstico, planificación, ejecución y eva- 
luación, el objeto de intervención del trabajador social en las instituciones educativas de la región Junín son los problemas y necesidades sociales de los estudiantes, porque en el trabajo cotidiano las trabajadoras sociales, intervienen permanentemente en los problemas y necesidades sociales de los estudiantes.

Las hipótesis especificas fueron: El objeto de intervención de la trabajadora social en las instituciones educativas de la región Junín son los problemas y necesidades sociales de los estudiantes, porque en el trabajo cotidiano las trabajadoras sociales, intervienen permanentemente en los problemas y necesidades sociales de los estudiantes; el objetivo de la intervención de la trabajadora social en las instituciones educativas de la región Junín es contribuir al bienestar social de los estudiantes; las funciones más frecuentes que cumple la trabajadora social en las instituciones educativas de la región Junín es el asistencial, gerencial, de educación social, de consejería -orientación social, seguido por la función de investigación; porque en el trabajo rutinario las trabajadoras sociales, por la naturaleza de la carrera, intervienen en diversas funciones; las formas más predominantes de intervención del trabajo social en las instituciones educativas de la región Junín es el individual, porque se interviene en el tratamiento de casos sociales de diversa índole y a la vez es grupal, porque los problemas sociales que se enfrenta se corrigen, en parte, en talleres educativos; el proceso metodológico de intervención del trabajador social en las instituciones educativas de la región Junín es investigación, diagnostico, planificación, ejecución, y evaluación.

\section{Metodología}

Tipo de investigación:
El tipo de investigación es básica, porque el estudio está orientado a conocer cómo es la intervención de la trabajadora social en las instituciones educativas.

\section{Nivel de investigación}

El nivel de investigación es el descriptivo, porque estuvo orientado a describir como es la intervención del trabajador social en las instituciones educativas de la región Junín.

\section{Diseño de investigación}

Es no experimental, transversal descriptivo. Es no experimental, porque el estudio se realizó en forma natural, sin manipular ninguna variable, tal como se presenta en la realidad; es transversal, porque el cuestionario se aplicó en un solo momento en tiempo único; es descriptivo, porque se describe como es la intervención de la trabajadora social en las instituciones educativas de la región Junín.

\section{Unidades de análisis, población y muestra}

Son los profesionales, trabajadoras sociales, que laboran en las instituciones educativas de la región Junín. La población en estudio es aproximadamente 15 trabajadoras sociales y la muestra fue la misma de la población.

\section{Método}

Se utilizó el método de análisis - síntesis; el análisis se realizó en el cuadro de operacionalizacion de variables, donde se disgrego la variable en dimensiones, indicadores y reactivos con el propósito de conocer la esencia del tema de investigación y el método de síntesis se realizó al presentar la demostración de las hipótesis y en la elaboración de las conclusiones.

\section{Operacionalización de variables}

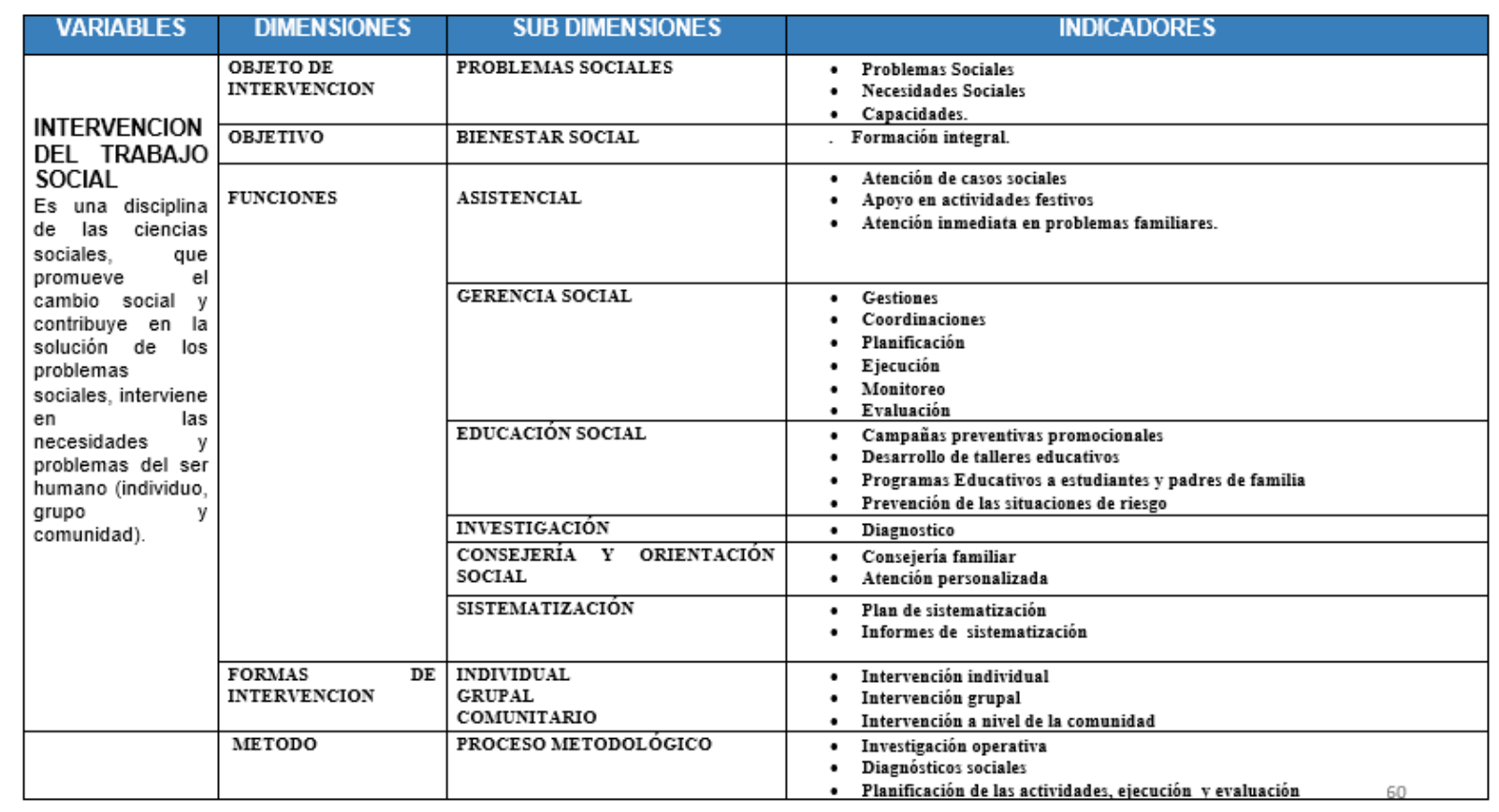




\section{Instrumento y técnica de investigación}

El instrumento fue el cuestionario y la técnica fue la encuesta aplicada a 10 trabajadoras sociales de las instituciones educativas de la región Junín.

Elaboración de los instrumentos; Para la recolección de datos se elaboró el cuestionario en base al cuadro de operacionalizacion de variables, una vez formulado se realizó la validación mediante juicio de expertos a 10 trabajadoras sociales.

Validación del instrumento; se realizó la validación con la aplicación de 6 expertos, y con el coeficiente de concordancia V de Aiken, resultando de 0.72 a 0.99 lo que indica que el instrumento es válido.

Aplicación del instrumento; validado el instrumento se aplicó a los trabajadores sociales que vienen laborando en las instituciones educativas de la región Junín, teniendo en cuenta que todas las preguntas sean respondidas

\section{Procesamiento de los resultados}

Aplicados los cuestionarios a la muestra de trabajadoras sociales, fueron procesados los resultados en el programa Excel para luego ser presentados en cuadros estadísticos.

\section{Resultados}

El objeto de intervención del trabajo social en las instituciones educativas de la región Junín fueron los problemas y necesidades de los estudiantes, siendo confirmado ello por las trabajadoras sociales en un $79 \%$; el $10 \%$ expresó que son los problemas sociales y el $11 \%$ restante, manifestó que el objeto de intervención son las capacidades de los estudiantes.

\section{Cuadro $\mathrm{N}^{\circ} 1$}

Objeto de intervención de las trabajadoras sociales de las instituciones educativas

\begin{tabular}{clcc}
\hline \hline $\mathbf{N}^{\circ}$ & \multicolumn{1}{c}{ ALTERNATIVAS } & FRECUENCIA & PORCENTAJE \\
\hline \hline 1 & $\begin{array}{l}\text { Problemas sociales de los } \\
\text { estudiantes }\end{array}$ & 1 & $10 \%$ \\
\hline \hline 2 & $\begin{array}{l}\text { Problemas y necesidades } \\
\text { sociales de los estudiantes }\end{array}$ & 12 & $79 \%$ \\
\hline \hline 3 & $\begin{array}{l}\text { capacidades de los estu- } \\
\text { diantes. }\end{array}$ & 2 & $11 \%$ \\
\hline \hline & TOTAL & $100 \%$ \\
\hline \hline
\end{tabular}

Nota: Cuestionario aplicado a las trabajadoras sociales de las instituciones educativas estatales de la región Junín

El Objetivo del trabajo social. - El $79 \%$ de las trabajadoras sociales manifestaron que es contribuir en la formación integral de los estudiantes de las instituciones educativas, seguido del $11 \%$ que consideran que el objetivo de intervención es contribuir al bienestar social de los mismos y el $10 \%$ expresa que es contribuir al fortalecimiento de las capacidades de dichos estudiantes.

\section{Cuadro $\mathrm{N}^{\circ} 2$}

Objetivos de intervención de las trabajadoras sociales de las instituciones educativas

\begin{tabular}{|c|c|c|c|}
\hline $\mathrm{N}^{\circ}$ & ALTERNATIVAS & FRECUENCIA & PORCENTAJE \\
\hline 1 & $\begin{array}{l}\text { Contribuir al bienestar so- } \\
\text { cial de los estudiantes de } \\
\text { las Instituciones educativas. }\end{array}$ & 3 & $20 \%$ \\
\hline 2 & $\begin{array}{l}\text { Contribuir en la formación } \\
\text { integral de los estudiantes } \\
\text { de las Instituciones Edu- } \\
\text { cativas.(calidad educativa } \\
\text { y valores) }\end{array}$ & 11 & $79 \%$ \\
\hline 3 & $\begin{array}{l}\text { Otro (Contribuir al fortaleci- } \\
\text { miento de las capacidades } \\
\text { básicas de los estudiantes a } \\
\text { fin de que sean protagonis- } \\
\text { tas de su propio desarrollo). }\end{array}$ & 1 & $1 \%$ \\
\hline & Total & 15 & $100 \%$ \\
\hline
\end{tabular}

Nota: Cuestionario aplicado a las trabajadoras sociales de las instituciones educativas particulares de la región Junín

Las Funciones más frecuentes que cumplen las trabajadoras sociales en las instituciones educativas de la región Junín es la asistencial, de educación social y la de gerencia social con un $100 \%$, seguido de la función de consejería y orientación social y la de investigación con $93 \%$. La función de sistematización no se realiza porque el resultado es $0 \%$.

\section{Cuadro $\mathrm{N}^{\circ} 3$}

Funciones que cumplen frecuentemente las trabajadoras sociales en las instituciones educativas

\begin{tabular}{clcc}
\hline \hline $\mathbf{N}^{\circ}$ & \multicolumn{1}{c}{ FUNCIONES } & FRECUENCIA & PORCENTAJE \\
\hline \hline 1 & Función Asistencial & 15 & $100 \%$ \\
\hline \hline 2 & Función de Investigación & 14 & $93 \%$ \\
\hline \hline 3 & $\begin{array}{l}\text { Función de Educación So- } \\
\text { cial }\end{array}$ & 15 & $100 \%$ \\
\hline \hline 4 & Función de Sistematización & 0 & $0 \%$ \\
\hline \hline 5 & $\begin{array}{l}\text { Función de Consejería y } \\
\text { Orientación Social }\end{array}$ & 14 & $93 \%$ \\
\hline \hline 6 & Función de Gerencia Social & 15 & $100 \%$ \\
\hline \hline & TOTAL & \\
\hline
\end{tabular}

Nota: Cuestionario aplicado a las trabajadoras sociales de las instituciones educativas particulares de la región Junín.

Las formas de intervención más frecuentes de las trabajadoras sociales, son el individual (Asesora y sugiere orientaciones y procedimientos a los actores de la comunidad educativa, aborda problemas específicos de los estudiantes como de adaptación, aprendizaje, conducta, rendimiento académico y otros) en un $100 \%$ y el grupal (reuniones de tutoría con los estudiantes, convivencia escolar, círculos de interaprendizaje, participación estudiantil, talleres de trabajo con padres de familia, talleres de capacitación a los docentes sobre clima escolar etc.) en un $100 \%$ en la práctica cotidiana de las trabajadoras sociales y la forma de intervención comunitaria es del 80 $\%$ (Escuela de padres, actividades orientadas.). 


\section{Cuadro $\mathrm{N}^{\circ} 4$}

Formas de intervención más predominantes de la trabajadora social en las instituciones educativas

\begin{tabular}{clcc}
\hline \hline $\mathbf{N}^{\circ}$ & \multicolumn{1}{c}{ ALTERNATIVAS } & FRECUENCIA & $\%$ \\
\hline \hline $\mathbf{1}$ & Individual & 15 & $100 \%$ \\
\hline \hline $\mathbf{2}$ & Grupal & 15 & $100 \%$ \\
\hline \hline $\mathbf{3}$ & Comunitario & 12 & $80 \%$ \\
\hline \hline $\mathbf{4}$ & Otros & 0 & 0 \\
\hline \hline
\end{tabular}

\section{TOTAL}

Nota: Cuestionario aplicado a las trabajadoras sociales de las instituciones educativas particulares de la región Junín

El proceso metodológico de la intervención profesional de las trabajadoras sociales en las instituciones educativas de la región Junín fue la investigación, diagnóstico, planificación, ejecución, monitoreo y evaluación de las actividades en un $80 \%$; el diagnóstico, planificación, ejecución y monitoreo es el $13 \%$ y el $7 \%$ considera que el proceso metodológico a trabajar es la investigación, diagnóstico y planificación y ejecución de las actividades.

\section{Cuadro $\mathrm{N}^{\circ} 5$}

Proceso metodológico de la intervención profesional de la trabajadora social en las instituciones educativas

\begin{tabular}{cccc}
\hline \hline $\mathbf{N}^{\circ}$ & ALTERNATIVAS & FRECUENCIA & $\%$ \\
\hline \hline $\begin{array}{l}\text { Investigación, diagnóstico } \\
\text { y planificación de las acti- } \\
\text { vidades }\end{array}$ & 01 & $7 \%$ \\
\hline \hline $\begin{array}{l}\text { Investigación, Diagnóstico } \\
\text {,planificación , ejecución, } \\
\text { monitoreo y evaluación }\end{array}$ & 12 & $80 \%$ \\
\hline \hline 3 & $\begin{array}{l}\text { Diagnóstico, planificación, } \\
\text { ejecución y Monitoreo. }\end{array}$ & 2 & $13 \%$ \\
\hline \hline & 15 & $100 \%$ \\
\hline \hline
\end{tabular}

Nota: Cuestionario aplicado a las trabajadoras sociales de las instituciones educativas particulares de la región Junín

En el Cuadro $\mathrm{N}^{\circ}$ 5, el $80 \%$ de las trabajadoras sociales mencionaron que dentro del proceso metodológico se incide más en la investigación, diagnóstico, planificación, ejecución, monitoreo y evaluación de las actividades; y en un $13 \%$, el diagnóstico, planificación, ejecución y Monitoreo, seguido del $7 \%$ que considera trabajar más con investigación, diagnóstico y planificación de las actividades.

\section{Demostración de la Hipótesis}

El objeto de intervención del trabajo social en las instituciones educativas de la región Junín son los problemas y/o necesidades sociales de los estudiantes de acuerdo al $79 \%$ de los trabajadores sociales, seguido de un 10\% que declaran que son las necesidades sociales; así mismo, un $7 \%$ manifiesta que únicamente son los problemas sociales de los estudiantes y el $11 \%$ expresó que son las capacidades. Con estos resultados se acepta la hipótesis específica uno.

El objetivo de intervención del trabajo social en las instituciones educativas de la región Junín, está centrado en la búsqueda del bienestar social de la población estudiantil; se rechaza esta hipótesis, porque se demuestra con la hipótesis alterna que, en un $79 \%$, las trabajadoras sociales manifiestan que el objetivo central de la intervención del trabajo social está orientado a la formación integral de los estudiantes de las instituciones educativas, basado en la calidad educativa y la práctica de valores. Con estos resultados se rechaza la hipótesis específica dos y se demuestra con la hipótesis alterna que el objetivo de intervención no es la búsqueda de bienestar social, sino la formación integral de los estudiantes.

Las funciones que cumplen frecuentemente el trabajo social en las instituciones educativas en la región Junín son: asistencial (atención inmediata y otros), gerencia (gestión, coordinación, planificación y organización), educación social (Talleres educativos), consejería y orientación social (casos sociales), seguido por la función de investigación social $93 \%$ y la función de sistematización no se cumple. Con estos resultados se acepta la hipótesis específica tres.

Las formas de intervención más predominantes del trabajo social en la instituciones educativas en la región Junín, es fundamentalmente grupal e individual en un $100 \%$, seguido por el comunitario en un $80 \%$; con estos resultados se confirma la hipótesis específica cuatro.

El proceso metodológico de la intervención del trabajo social en las instituciones educativas en la región Junín fue la investigación diagnóstica, planificación, ejecución y evaluación de las actividades en un $80 \%$, seguido por el diagnostico planificación ejecución y monitoreo en $13 \%$ y finalmente investigación, diagnóstico y planificación de las actividades en un 7\%. Con estos resultados se acepta la hipótesis específica cinco.

La intervención del trabajo social en las instituciones educativas en la región Junín, es fundamentalmente asistencial, de gerencia social y de educación social, seguido en un $100 \%$ por consejería y orientación, el objeto de intervención son los problemas y necesidades sociales. El objetivo es contribuir a la formación integral de los estudiantes basados en la calidad educativa y de valores; las formas de intervención predominantes es el grupal e individual seguido por la forma de intervención comunal, continuando con el proceso metodológico de investigación, diagnóstico, planificación, ejecución, monitoreo y evaluación. Con estos resultados se acepta la hipótesis general.

\section{Discusión}

El objeto de intervención del trabajo social en las instituciones educativas de la región Junín son los problemas y/o necesidades sociales de los estudiantes de acuerdo al 
$79 \%$ de los trabajadores sociales, seguido de un 7\% que manifiestan que son las necesidades sociales; así mismo, un $7 \%$ declara que únicamente son los problemas sociales de los estudiantes.

Estos resultados se sustentan en Aguilar (2013), en el estudio Trabajo social, concepto y metodología, cuando manifiesta que

en el ámbito del trabajo social y siempre a partir de la práctica pre profesional de ayuda, todas las cuestiones relativas al objeto de estudio e intervención se ha planteado en torno a las necesidades... (Pg. 25)

Es decir, de acuerdo al desarrollo histórico de la carrera se han ido introduciendo diversas modificaciones en el objeto de intervención de la carrera, así por ejemplo, hay una corriente de pensamiento que establece como objeto del trabajo Social las necesidades sociales y su satisfacción. Además plantea, como objeto del trabajo social, la persona en situación con problema, las capacidades del ser humano entre otros. Estas propuestas evidentemente se producen por las diversas concepciones y por la falta de una teoría rigurosamente sistemática.

Además, Aylwin (s/f), en el estudio El objeto del trabajo Social, menciona que

pocas nociones son tan familiares al trabajo social como la del problema social, profesión nacida justamente para enfrentar científicamente los problemas sociales, el trabajo social encuentra en ellos su materia prima, su objeto de acción. Su centro de interés está constituido por el estudio y tratamiento de los problemas sociales. (Pg. 6)

Por otra parte, Maya (2008), sustenta en el estudio Objeto y modo de actuación del trabajo social, que:

El trabajo social interviene mediante una acción planificada en la solución de los problemas sociales específicos que afectan a las personas, grupo, organizaciones, comunidades y sectores sociales...

En este sentido y en concordancia con el carácter complejo, multidimensional, global, y contextual que asume "lo social" como objeto de intervención del trabajo social, su modo de actuación tendrá necesariamente un enfoque multidisciplinario, fundamentado en el acervo teórico y métodos propios del trabajo social, construidos desde un enfoque transdisciplinario. Sin embargo, cuando se refiere a "lo social" y o los problemas sociales, los teóricos consideran que no es propiedad de la disciplina concreta porque las cuestiones relativas a la vida social y a la acción social se extienden a todas las disciplinas científicas y humanas. Campos (2008), citado por Tibaria y Rico (2009: pág.; /54).

Por otra parte, Tobón, Rottier y Manrique (1998), fundamentan en La práctica profesional del trabajo social. Guía de análisis CELATS Argentina, que referido al obje- to de intervención, es preciso tener presente que en la intervención debe hacerse una clara distinción entre el diagnostico que se realiza de la situación que se presenta como problemática y la definición de ésta en términos de intervención propiamente dicho; es decir, que definir el problema objeto de intervención no es de ninguna manera detectar el problema del sujeto social "es delimitar de una necesidad social si son susceptibles de modificar con nuestra intervención"; visto de esta manera, el objeto de intervención puede ser definida como la esencia de la profesión que representa un situación social, la cual es abordada desde una interpretación subjetiva con características definidas que pueden ser cambiantes y continuos.

El objetivo de la intervención del trabajo social en las instituciones educativas de la región Junín está orientado a la formación integral de los estudiantes según el $79 \%$ de las trabajadoras sociales encuestadas, seguido del 20 $\%$ que respondieron que el objetivo es contribuir bienestar social de los estudiantes y el $1 \%$ que expresa que es contribuir al fortalecimiento de las capacidades básicas de los estudiantes.

Estos resultados se sustentan en Murcia, Martínez y Ortíz (2007), en el estudio La intervención del trabajo social desde la práctica de entretenimiento profesional en instituciones educativas, cuando dicen, que

dependiendo del tipo de institución, la intencionalidad de la intervención social adquiere diferentes sentidos, el principal objetivo de las instituciones es lograr el desarrollo integral del educando, y la institucionalizada que devela el profesional en su actuar, tiene a los objetivos profesionales y se fundamenta en los conceptos de desarrollo integral, formación integral y valores. (pág. : 84,85).

Así mismo, se sostiene en el Manual de la Organización de la Escuela de Educación Secundaria Técnica (1982:85), citado por Amador (2002), que la intervención de la trabajadora social en las instituciones educativas de educación secundaria tiene como propósito "coadyuvar a la formación integral del educando en su proceso de adaptación al medio ambiente escolar, social y económico en que se desarrolla” (pág. 104)

Las funciones que cumple frecuentemente el trabajo social en las instituciones educativas en la región Junín son: asistencial (atención inmediata y otros), administrativa (gestión y planificación), educación social (talleres educativos), consejería y orientación social (casos sociales) en un $100 \%$, seguido por la función de investigación en un $93 \%$.

Fernández y otros (2002), citados por Murcia, Martínez y Ortiz (2008), en el estudio Intervención del trabajo social en el área educativa en 14 colegios de Bogotá, sostienen

que el rol del trabajador social gira en torno a asumir funciones de tipo administrativo; es decir, de 
organización y prestación de un servicio y de tipo operativo en la atención directa a los sujetos de intervención (estudiantes, familias, servicios y docentes.

Igualmente en Álvarez y otros, en el estudio Competencias de los y las trabajadoras sociales en el aspecto educativo en instituciones de secundaria, evidencian como el trabajo social contribuye a la construcción del PI aportado en diagnóstico, una planeación y una gestión de proyecto que evidencia las necesidades y recursos existentes en la institución educativa (pág.: 19). Así mismo, Amador (2007), sustenta en el estudio titulado La intervención del trabajador social en los conflictos escolares que se presentan en el nivel secundario;

que los trabajadores sociales para poder desarrollar su quehacer profesional requieren el ejercicio de funciones, las cuales dependerán de los contextos en los que se encuentran inmersos; es decir, pueden orientarse a la planificación y programación, a la administración; así como, a la investigación, gestión, educación, organización y capacitación social. (Pág. 29)

Por otra parte, Cajamarca (2015), en el estudio El trabajo social y la educación de Bachillerato General Unificado (BGU), menciona "que las funciones del trabajo social son preventivas, de atención, coordinación mediación, seguimiento, supervisión, evaluación, capacitación e investigación.” (Págs.: 34, 40, 41); asímismo, Ander Egg (1996), menciona que las funciones específicas del trabajo social son las siguientes: gestor y mediador entre personas e instituciones; asesor, orientador y consejero social; investigador de problemas, necesidades y conflictos sociales; identificador de recursos y potencialidades para la acción; movilizador de recursos internos y externos como planificador de tratamientos y evaluador de servicios y programas.

Por otra parte, se considera necesario señalar que la carrera de trabajo social no es valorado por el resto de disciplinas de las ciencias sociales, debido a que muchas veces el profesional, trabajador social, es visto como un gestionador de recursos y no tiene mayor desempeño o sustento teórico, entonces es conminado a tareas rutinarias y administrativas.

Las formas de intervención más predominantes del trabajo social en la instituciones educativas de la región Junín es fundamentalmente grupal e individual en un 100 $\%$, seguido por el comunitario en un $80 \%$.

Estos resultados se sostienen en Amador (2007), quien en su estudio La intervención del trabajo social en los conflictos escolares que se presentan en el nivel secundario, Universidad de Colima, manifiesta que

el trabajo social en el ámbito educativo se enfoca en la intervención familiar, grupal, comunitaria a diferencia de los otros profesionales quienes intervienen de manera individual enfocándose en gran medida en las dificultades propias de cada individuo y no en los factores del contexto social que provocan serios problemas a los estudiantes.

Además, en la Agenda nacional de evaluación de la calidad y acreditación (2005), citado por Alvarado y Granados (2008), se explica

que el trabajo social, como disciplina, aplica la metodología especifica en la que se integra el trabajo social de caso, grupo y comunidad.

El proceso metodológico de la intervención del trabajo social en las instituciones educativas de la región Junín es la investigación diagnóstica, planificación, ejecución y evaluación de las actividades en un $80 \%$, seguido por el diagnostico planificación ejecución y monitoreo $13 \%$ y finalmente investigación, diagnóstico y planificación de las actividades un $7 \%$.

Estos resultados se sustentan en Cajamarca (2015), en el estudio Trabajo social y educación de bachillerato general unificado, cuando manifiesta que

el proceso metodológico de los profesionales depende más de los lineamientos institucionales que de una metodología común en el campo, aunque desarrollen elementos comunes como la atención individual y el seguimiento de caso. Algunas profesionales retoman para su actuar procesos lógicos de diagnóstico, planeación y ejecución con la ayuda de instrumentos para realizar la acción y evaluar los resultados esperados. (pág., 85).

Además, se sustenta en Aguilar (2013), que

el proceso metodológico de intervención del trabajo social es el estudio, diagnostico, programación, ejecución y evaluación. (pág.; 278).

La intervención del trabajo social en las instituciones educativas de la región Junín es fundamentalmente administrativa, asistencial, de educación social, consejería y de orientación social en un $100 \%$, el objeto de intervención son los problemas y necesidades sociales, el objetivo es contribuir a la formación integral de los estudiantes; las formas de intervención predominantes es el grupal e individual seguido por la forma de intervención comunal, a través de un proceso metodológico de investigación, diagnóstico, planificación, ejecución y evaluación.

Estos resultados se sustentan en Barros (1976), citado por Aylwin (s/f), cuando relaciona la noción de problema con la necesidad, al afinar que el problema social es la manifestación de una carencia que afecta a sectores considerables de la población, impidiéndoles satisfacer sus necesidades básicas y lograr el pleno desarrollo de sus potencialidades.

Además, se sostiene en Murcia, Martínez y Ortiz (2007), 
en el estudio Intervención del trabajo social desde la práctica de entrenamiento profesional en instituciones educativas, dice, que

dependiendo del tipo de institución, la intencionalidad de la intervención social adquiere diferentes sentidos, el principal objetivo de las instituciones es lograr el desarrollo integral del educando, y la institucionalizada que devela el profesional en su actuar, tiene a los objetivos profesionales y se fundamenta en los conceptos de desarrollo integral, formación integral y valores (pág.: 84,85).

Por otra parte, Fernández y otros (2002), en el estudio Intervención del trabajo social en el área educativa en 14 colegios de Bogotá, sostienen

que el rol del trabajador social gira en torno a asumir funciones de tipo administrativo; es decir, de organización y prestación de un servicio y de tipo operativo en la atención directa a los sujetos de intervención (estudiantes, familias, servicios y docentes). Igualmente, Álvarez y otros, en el estudio Competencias de los y las trabajadoras sociales en el aspecto educativo en instituciones de secundaria cuando evidencian como el trabajo social contribuye a la construcción del PI aportado en diagnóstico, una planeación y una gestión de proyecto que evidencia las necesidades y recursos existentes en la institución educativa (pág. : 19).

Así mismo, estos resultados se sostienen en Amador (2007), en su estudio La intervención del trabajo social en los conflictos escolares que se presentan en el nivel secundario, cuando manifiesta que

el trabajo social, en el ámbito educativo, se enfoca en la intervención familiar, grupal y comunitaria a diferencia de los otros profesionales quienes intervienen de manera individual enfocándose en gran medida en las dificultades propias de cada individuo y no en los factores del contexto social que provocan serios problemas a los estudiantes.

Finalmente, Aguilar (2013), expresa que el proceso metodológico de intervención del trabajo social es el estudio, diagnóstico, programación, ejecución y evaluación. (pág.; 278).

\section{Conclusiones}

El objeto de intervención del trabajo social en las instituciones educativas de la región Junín, son los problemas, necesidades sociales de los estudiantes, susceptibles de ser abordados con nuestra intervención mediante una acción planificada en la solución de problemas específicos.

El objetivo de la intervención del trabajo social en las instituciones educativas de la región Junín, es contribuir a la formación integral de los estudiantes, se fundamenta en el desarrollo integral, formación integral y valores.
Las funciones que cumple frecuentemente el trabajo social en las instituciones educativas de la región Junín es de gerencia social, asistencial, educación social, consejería y orientación, seguido por la función de investigación y la función de sistematización no se realiza.

Las formas de intervención más frecuentes del trabajo social en las instituciones educativas de la región Junín es el grupal e individual, seguido por el comunitario porque en la cotidianidad el trabajo social interviene más en el trabajo de grupos, individual (casos sociales y grupos). El proceso metodológico de la intervención del trabajo social en las instituciones educativas de la región Junín es la investigación, diagnostico, planificación, ejecución y evaluación de los resultados esperados.

La intervención del trabajo social en las instituciones educativas de la región Junín es fundamentalmente administrativo, asistencial, de educación social, consejería y orientación social; el objeto de intervención son los problemas y necesidades sociales, el objetivo es contribuir a la formación integral de los estudiantes; las formas de intervención predominante es el grupal e individual seguido por la forma de intervención comunal a través de un proceso metodológico de investigación diagnóstico, planificación, ejecución y evaluación.

\section{Recomendaciones}

Se recomienda investigar sobre las funciones más predominantes en el ejercicio de la carrera desde el enfoque cualitativo, con el objetivo de contribuir e identificar las funciones que se cumple en la actualidad.

Se sugiere realizar estudios sobre cómo es la formación profesional y qué relación tiene con la intervención profesional del trabajo social en el sector educación.

Se recomienda investigar sobre el modelo del acompañamiento de los estudiantes a través de la acción tutorial integral, que es un modelo reciente del servicio educativo que busca mejorar la calidad de servicio de la educación secundaria, emitido en mayo del 2017.

\section{Referencias bibliográficas}

Amador J. (2007) La intervención del trabajo social en los conflictos escolares que se presentan en el nivel secundario, Universidad de Colima Facultad de Trabajo Social. México.

Aguilar, M.(2013) Trabajo Social, concepto y metodología , consejo general del Trabajo Social, Editorial Paraninfo. España.

Ander -Egg (2009) Introducción al Trabajo Social, Buenos Aires Argentina. 02/02/2017. Recuperado de: https: / / trabajosocialudla.files.wordpress.com/.../ 
introduccion-al-trabajo-social-ezequiel.

Aylwin N., (1980) El objeto del trabajo Social - artículo de revista de trabajo Social. Recuperado en 25/10/2017. https://repositorio.uc.cl/bitstream/handle/11534/6194/000379758.pdf?sequence $=1$

Barranco, C. y López G. (2009). El sentido del Trabajo Social en Educación: reflexiones sobre la experiencia profesional en los EOEPS, España.

Ballesteros A., Vizcarret, J.,Uriz, M., (2013) Funciones profesionales de los trabajadores sociales en España, Cuadernos de trabajo Social. Vol.26-I (2013) 127-138, ICCN: 0214-0314.

Beltrán E., Jhon E. (2010) Reflexiones en torno al debate formativo y profesional, Universidad Nacional de Colombia.

Córdova S. y Patricia (2011) El trabajo Social en el Chile del siglo XXI, satisfacciones y descontentos y desafíos en la ejecución de la profesión, Santiago de Chile.

Cajamarca F. (2015) El trabajo social y la educación del bachillerato general unificado, Universidad de Cuenca, Facultad de Jurisprudencia, Escuela de Trabajo Social y Orientación Familiar. Cuenca Ecuador .

Castro C. G.; Chávez C. y Vásquez G. (2014) Epistemología y trabajo social, Universidad Autónoma de Sinaloa.

ESPINOSA, M., (1990) Análisis de funciones del Trabajador Social en el campo educativo, España.

Flores S. C. (s/f) Hacia una concepción del trabajo Social contemporáneo en México, Universidad Autónoma de México.

Fernández R., y otros (2002) Intervención del trabajo Social en el Área Educativa en 14 colegios de Bogotá, Universidad Monserrat.

Maya, E.,(2008) Objeto y modo de actuación del Trabajador Social, Boletín electrónico Surá N\#154, Escuela del Trabajo Social Universidad de Costa Rica. Recuperado en 25/10/2017 http://unpan1.un.org/ intradoc/groups / public/documents/icap/unpan048763.pdf

Murcia D., Martínez V. y Ortiz J. (2007) La intervención del trabajo social desde la práctica de entretenimiento profesional en instituciones educativas, Facultad de Trabajo Social, Universidad de la Salle.
Pedraza J., Vela A. y Sicail J. (2006) La intervención del trabajo social en programas educativos de la región Junín Universidad de la Salle.

Salvador E.(2010) Procesos de intervención profesional en instituciones educativas de nivel maternal, inicial y primaria, revista de Trabajo Social - FCH- UNCPBX.

Silva, L.,(1996) Teoría del trabajo social y práctica de comunidad: Un análisis del método básico de Ma. Angélica Gallardo ClarK, México

Tibana, D; y Rico J. (2009) Fundamentación de la Intervención del trabajo social. Sistema conceptual y avances, Universidad de la Salle, Facultad de Ciencias Económicas y Sociales, programa de Trabajo Social, Bogotá.

Tobón M., Rottier N.,y Manrique A., (1998) La práctica profesional del trabajo social. Guía de análisis - CELATS Editorial Humanitas Argentina. Recuperado 25/10/2017 http://www.academia. edu/8475817/la_practica_profesional_del_trabajador_social

Him A. (2013) El quehacer de los trabajadores sociales de educación del control del ausentismo a la inclusión educativa. Universidad Nacional de la Plata Argentina. 